\title{
Procédé de superfinition par toilage : analyse énergétique des variables «process»-temps de cycle et fréquence d'oscillation
}

\author{
Patrick Ghidossi $^{1, a}$, Mohamed El Mansori ${ }^{1}$, Edoardo Sura ${ }^{2}$, Régine Geoffroy ${ }^{1}$ \\ et StÉphane Deblaise ${ }^{2}$ \\ 1 Laboratoire de Mécanique et Procédés de Fabrication (LMPF-JE2381), ENSAM, rue Saint Dominique, BP 508, \\ 51006 Châlons-en-Champagne, France \\ 2 Renault, Direction de l'Ingénierie Mécanique, 92508 Rueil-Malmaison Cedex, France
}

Reçu 23 janvier 2007, accepté le 23 janvier 2007

\begin{abstract}
Résumé - Les succès des applications de superfinition par toilage ont été longtemps attribués à l'art d'appliquer des combinaisons variées de conditions et de séquences, pour un dispositif de toilage particulier, sans aucune méthode apparente établie. Du point de vue laïque ceci pourrait sembler vrai. Toutefois il y a des mécanismes physiques bien définis qui sont essentiellement de nature fondamentale. Une description énergétique du procédé de toilage est introduite. La variable d'énergie dissipée est considérée pour étudier l'effet du temps de toilage et observer l'influence supposée d'« alignement » (oscillation axiale de la bande abrasive). Deux régimes de toilage sont identifiés et ainsi discutés.
\end{abstract}

Mots clés : Toilage / usure / abrasion / approche énergétique/ rugosité

\begin{abstract}
Belt grinding process: an energetic approach to cycle-time and oscillation frequency effects. Successful applications in superfinishing by belt grinding have long been attributed to the art of applying various combinations of conditions and sequences for a particular set-up without any apparent established method. From the layman's point of view, this might appear to be true. However, there are very definite physical mechanisms which are essentially fundamental in nature. An energy description of the belt grinding process is introduced. The dissipated energy variable is considered to investigate the timegrinding dependant behavior and to observe the possible influence of "tracking" (axial belt oscillation). Two "belt grinding regimes" are identified and then discussed.
\end{abstract}

Key words: Belt grinding / wear / abrasion / energy approach / roughness

\section{Introduction}

L'usinage de précision de surfaces fonctionnelles de pièces mécaniques, c'est-à-dire l'obtention de pièces sans défaut sur le plan géométrique, uniquement par le biais des procédés de pré-usinage (tournage et rectification) nécessite un effort technico-économique considérable. Ces procédés sont par conséquent complétés par des procédés avancés d'abrasion, dits de superfinition, tels que la rectification à traits croisés, le rodage à course longue, le toilage ... L'objectif est de réduire voire de supprimer les imperfections sur la surface de la pièce et dans la structure du matériau dues au procédé de pré-usinage.

\footnotetext{
a Auteur pour correspondance :

patrick.ghidossi@chalons.ensam.fr
}

Le toilage est une variante de ces procédés de finition avancés dont la particularité réside dans sa neutralité sur le plan thermique. On part généralement de pièce pré-usinée par tournage et rectification, sur laquelle on imprime un mouvement d'oscillation et de rotation, que l'on presse à froid contre une toile abrasive. La surface qui en résulte est exempte des tensions des surfaces en sous-couches ou des microfissures. La structure d'origine est ainsi dégagée et la fonctionnalité de surface sous une forte contrainte est nettement améliorée. Dans son principe, ce procédé de finition est remarquablement simple et peu coûteux. Malheureusement, sa maîtrise sur le plan industriel est loin d'être acquise. Ainsi, la mise au point d'une gamme de toilage reste encore empirique, issue d'un ensemble de règles pratiques, qui s'apparentent plutôt à des « recettes ». Pour preuve, chaque nouvelle spécification 


\section{Nomenclature}

\begin{tabular}{|ll|}
\hline$E_{\text {toilage }}:$ & énergie absorbée par l'opération de toilage $(\mathrm{J})$ \\
$f:$ & fréquence d'oscillation du contact toile-pièce $(\mathrm{Hz})$ \\
$G_{R}:$ & rapport du critère de rugosité $R$ avant et après l'opération de superfinition \\
$\mathrm{N}:$ & vitesse de rotation de la pièce $\left(\operatorname{tr} \min ^{-1}\right)$ \\
$P_{0}:$ & puissance à vide $(\mathrm{W})$ \\
$P_{\text {charge }}:$ & puissance en charge $(\mathrm{W})$ \\
$P_{\text {toilage }}:$ & puissance absorbée par l'opération de toilage $(\mathrm{W})$ \\
$R:$ & critère de rugosité conformément à la norme NF EN ISO $12085(\mu \mathrm{m})$ \\
$t_{\text {toilage }}:$ & durée de l'opération de toilage $(\mathrm{s})$ \\
\hline
\end{tabular}

d'état de surface sur un plan-produit se traduit, pour le process, par un travail important de codification de règles proposées sous forme de procédures et de tolérances, indispensables à une fabrication en série. Il est clair que, dans ce domaine, le lancement de campagnes d'essais à chaque nouvelle spécification avec des coûts de fabrication très élevés réduit les ressources projets et occasionne quelquefois des risques d'investissements inadaptés. L'apport de la physique de l'abrasion permettrait sans doute de diminuer substantiellement les coûts de cette phase initiale et de rationaliser la production en série. Ceci est d'autant plus vrai que les variables « process » d'influence sont extrêmement nombreuses, qu'elles s'interpénètrent de façon fort complexe et que, en somme, les études réellement nouvelles intervenues scientifiquement depuis plusieurs décades sont quasi-inexistantes. Il suffit de regarder les dates auxquelles ont été publiées et proposées les définitions et règles qui régissent en partie les pratiques industrielles [1]. De plus, il n'a pas été trouvé dans la littérature d'études récentes et scientifiques traitant directement des procédés de superfinition par toilage et notamment de la technologie IMPCO utilisée par l'alliance RENAULT-NISSAN. Il existe sûrement quelques données et savoir-faires non publiés et propres à certaines entreprises de mécanique utilisant cette variété de procédés de finition avancés. En revanche, il existe de nombreuses publications sur l'abrasion à deux corps ou trois corps. Malheureusement leurs résultats n'ont pas pu être extrapolés au cas du toilage car les conditions de sollicitations sont trop différentes. En effet, dans le cas du pierrage, des travaux menés par Puthanangary et al. [2], Varghese et al. [3], Chang et al. [6], ont précisé l'influence prépondérante de certains paramètres tels que la pression de contact, le temps de cycle et la fréquence d'oscillation sur le taux d'enlèvement de matière. Les effets sur la rugosité de surface obtenue et l'énergie spécifique de coupe impliquée ont été également analysés [5]. Certains auteurs [6] ont synthétisé les résultats expérimentaux obtenus sous forme de «carte de process ». Quelques tentatives de modélisation des phénomènes mis en jeu en pierrage ont été proposées [4]. Elles sont basées essentiellement sur une approche statistique du contact abrasif/surface usinée, mais ne tiennent pas compte des phénomènes d'usure. Aussi, les phénomènes physiques impliqués tels que la coupe, la déformation plastique (labourage) et le frottement ne sont pas pris en compte.
L'objectif de cette étude est de comprendre le procédé du toilage (technologie IMPCO) en s'intéressant aux mécanismes physiques qui gouvernent les interactions du contact oscillant film abrasif/pièce. La démarche expérimentale préconisée ici pour étudier le contact tribologique oscillant film abrasif/pièce en toilage s'apparente à celle utilisée en pierrage [2]. Un banc d'essai de toilage sur pièces réelles avec un bras d'usinage instrumenté a été ainsi développé. En effet, les mesures des valeurs effectives des efforts de serrage, des énergies élémentaires mises en jeu (via des mesures de puissance différenciées) dans la zone d'usinage font partie des informations indispensables, dans un premier temps, à la description approchée du tribosystème toile/pièce et à la mise au point, après expertise, d'essais spécifiques de compréhension mieux adaptés.

\section{Démarche expérimentale}

\subsection{Dispositif de toilage}

Le banc d'essai (cf. Fig. 1) consiste en un tour conventionnel équipé d'un appareil de superfinition de type Bader avec un bras d'usinage. Ceci conduit à une architecture horizontale de superfinition par toile; architecture habituellement utilisée pour la superfinition de portées cylindriques (vilebrequins). Le banc de toilage dispose d'une puissance de $9 \mathrm{~kW}$ à la broche de la machine-outil. Afin de reproduire au mieux les conditions de superfinition de portées cylindriques, les essais ont été réalisés sur des pièces à paliers successifs. Elles sont cylindriques de $54,780 \mathrm{~mm}$ de diamètre et composées de 5 paliers de $30 \mathrm{~mm}$ de long (voir Fig. 1). Le matériau est un acier faiblement allié 42 Cr Mo4 traité thermiquement pour atteindre une dureté de 55-60 HRc. Les pièces sont usinées par tournage dur (tournage après traitement thermique) avec un outil en carbure de tungstène de type K10. La toile de $20 \mathrm{~mm}$ de large est constituée de grains abrasifs d'oxyde d'alumine de taille moyenne d'environ $30 \mu \mathrm{m}$. Elle est pressée par vérin pneumatique sur la périphérie du palier usiné par l'intermédiaire de deux mâchoires d'ébauche pendant une durée prédéfinie $t_{\text {toilage }}$ Chacune des mâchoires est constituée de 5 pierres (grain corindon) assurant l'enroulement et le contact de la toile sur la surface du palier, en couvrant un angle de $160^{\circ}$. L'effort de 


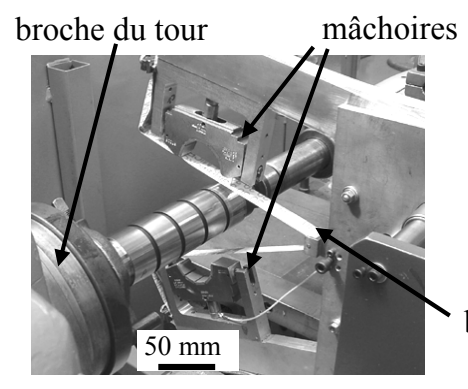

Mâchoires ouvertes

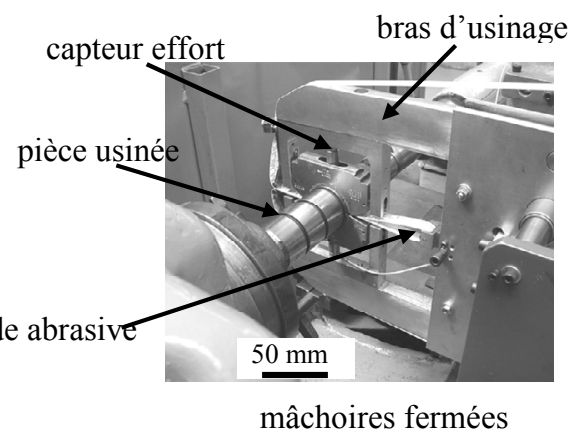

Fig. 1. Dispositif de toilage avec bras d'usinage.

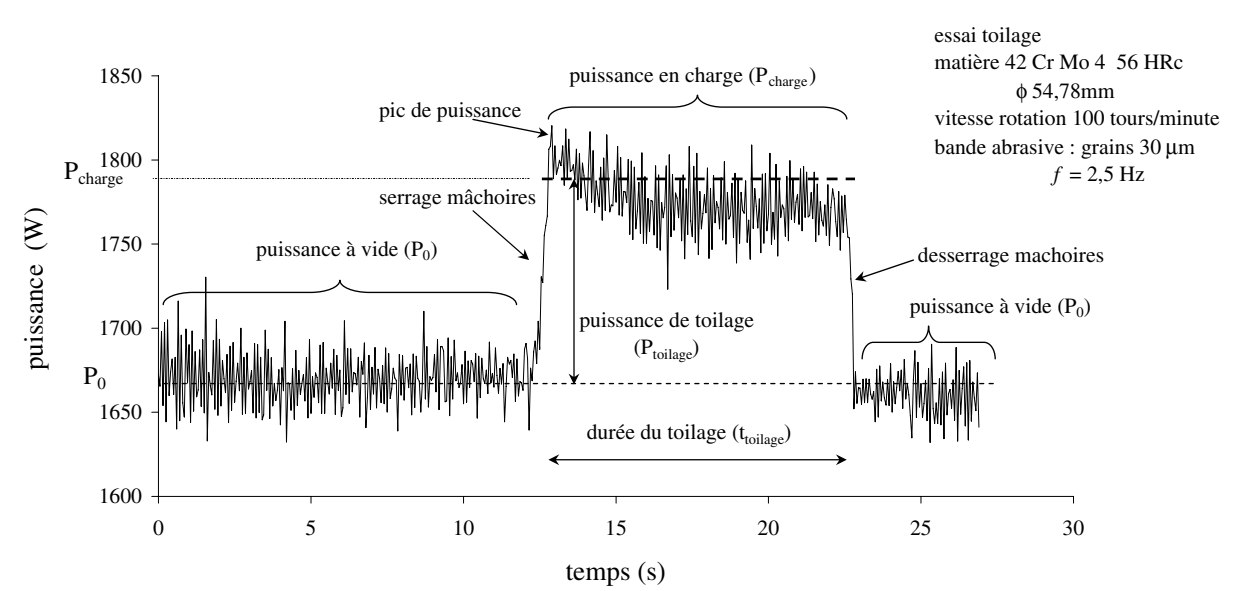

Fig. 2. Courbe typique de l'évolution de la puissance consommée au cours d'une opération de toilage.

serrage de chacune des mâchoires est de 400 N. Il est mesuré par un capteur piezo-électrique Kistler type 9011A. L'enlèvement de matière est assuré par deux mouvements combinés de rotation du cylindre usiné ( $N$ tours par minute) et d'oscillation des deux mâchoires comprimant la bande abrasive (dans le sens axial de la pièce, à la fréquence d'oscillation $f$ ).

Deux campagnes d'essais ont été réalisées :

- la première explicite le mode d'intervention de la variable « process » durée de superfinition ou temps de cycle : $t_{\text {toilage }}$;

- la seconde montre l'influence de la fréquence d'oscillation du contact toile-pièce : $f$.

Tous les essais de toilage ont été réalisés à sec sans avance de toile. La puissance absorbée par le processus de toilage est évaluée par la différence entre la puissance en charge $P_{\text {charge }}$ et celle à vide $P_{0}$ de la machine-outil (tour). Son acquisition in situ s'effectue par le biais d'un transducteur de puissance DigitalWay - $16 \mathrm{~kW}$ associé au logiciel de traitement Tool Power Analyser. L'analyse de la rugosité obtenue en fonction des différents paramètres technologiques s'appuie sur des mesures d'états de surface (sur profilomètre 3D Surfascan) avant et après toilage. Elle est complétée par des observations au microscope électronique à balayage (M.E.B.). Chaque essai de toilage est répété 5 fois pour permettre une analyse statistique.

\subsection{Résultats expérimentaux}

\subsubsection{Résultats des tests énergétiques}

Les courbes de variation de la puissance en charge et à vide en fonction du temps définissent la valeur de la puissance absorbée par le process de toilage $P_{\text {toilage }}$ (Fig. 2). Celle-ci s'ajoute à la puissance à vide $P_{0}$ dès le serrage des mâchoires, puis progresse en quelques secondes jusqu'à un maximum. Elle décroît ensuite pour se stabiliser pendant le reste de la durée de toilage $t_{\text {toilage }}$. Le pic observé dans l'évolution de la puissance en charge se trouve parfois occulté par le temps nécessaire à la fermeture complète des mâchoires.

Les calculs de la puissance absorbée par le toilage $P_{\text {toilage }}$ en fonction de la durée de l'opération $t_{\text {toilage }}$ sont reportés sur la figure 3 . Il est constaté que celle-ci diminue si $t_{\text {toilage }}>t_{\mathrm{c}}$ et croît si $t_{\text {toilage }}<t_{\mathrm{c}}$. La valeur $t_{\mathrm{c}}$ marque le passage à un régime énergétique qui minimise la puissance absorbée par le processus de toilage. Le test statistique d'Aspin-Welch et Student [7] confirme la chute significative de puissance absorbée au risque de $1 \%$ entre $t_{\text {toilage }}=3 \mathrm{~s}$ et $t_{\text {toilage }}=12 \mathrm{~s}\left(t_{\text {student }}=5,95 ; t_{1 \%}=3,71\right)$. Sur la figure 4 est reportée l'évolution de la puissance de toilage $P_{\text {toilage }}$ en fonction de la fréquence d'oscillation $f$. La durée de l'opération de toilage $t_{\text {toilage }}$ est fixée pour $t_{\text {toilage }}>t_{\mathrm{c}}\left(\right.$ dans ce cas $\left.t_{\text {toilage }}=9 \mathrm{~s}\right)$. Aucune variation significative n'est constatée lorsque la fréquence d'oscillation varie de 0 à $3,33 \mathrm{~Hz}$. Toutefois, des dispersions 


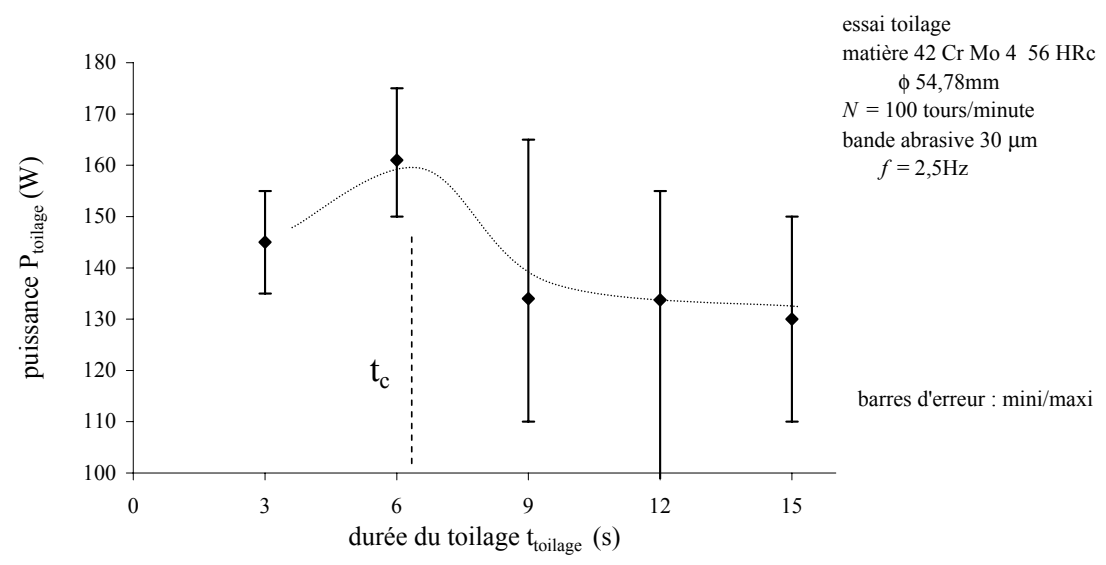

Fig. 3. Évolution de la puissance de toilage $P_{\text {toilage }}$ en fonction de $t_{\text {toilage }}$.

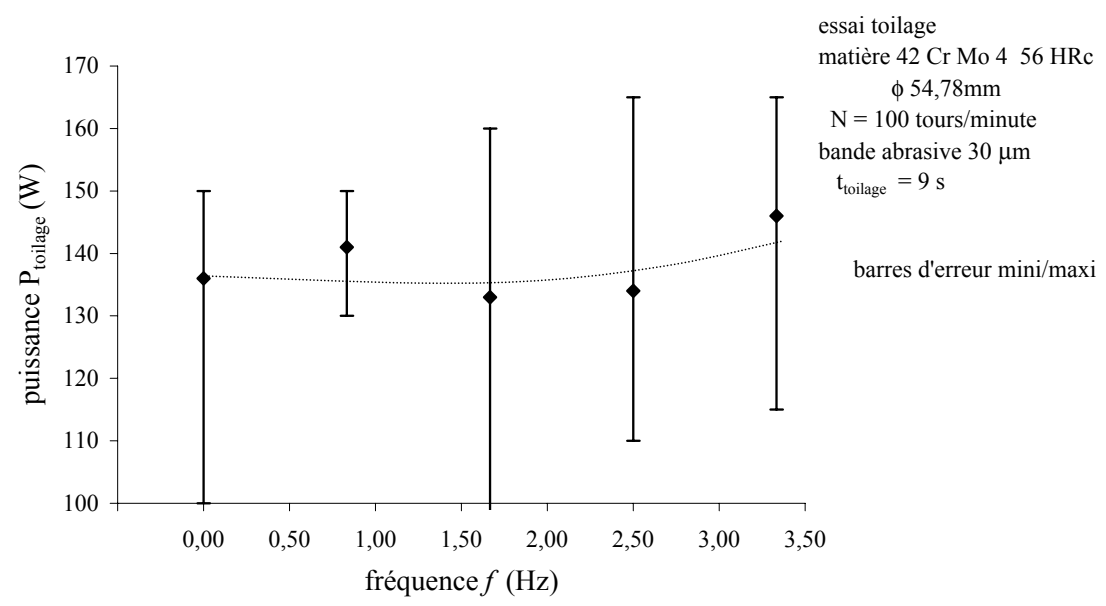

Fig. 4. Puissance de toilage $P_{\text {toilage }}$ en fonction de la fréquence d'oscillation $f$.

importantes sur toute la plage de fréquences étudiée perturbent en effet l'analyse. Ces dernières peuvent s'expliquer ainsi :

- il est clair que le critère intrinsèque de puissance absorbée ne suffit pas à caractériser finement l'influence de la variable fréquence. Ce critère globalise en effet les phénomènes physiques (coupe, déformation plastique et frottement) ;

- des dispersions intrinsèques aux durées programmées $t_{\text {toilage }}$ des essais sont inévitables. Le détail de ces variations en fonction de la durée $t_{\text {toilage pour chaque }}$ essai est donné dans la figure 5.

Une analyse énergétique supplémentaire basée sur l'énergie de toilage $E_{\text {toilage a été effectuée. La figure } 6}$ représente la variation de celle-ci en fonction de la durée effective d'usinage. Dans cette représentation, on distingue aussitôt deux régimes de toilage :

- pour $t<t_{\mathrm{c}}$, un régime de toilage primaire où le processus de toilage est dominé par la formation de microcopeaux, mais au prix d'énergies dissipées élevées $\left(E_{\text {toilage }} \approx 550\right.$ joules $)$

- pour $t>t_{\mathrm{c}}$, un régime de toilage secondaire (régime établi) où l'opération de toilage s'apparente alors plus à un frottement qu'à un enlèvement de matière (l'énergie de toilage $E_{\text {toilage }}$ chute à 300 joules environ).

\subsubsection{Interprétation des tests énergétiques}

\section{a) État de surface}

Il est clair que l'état de surface finie représente l'aboutissement corrélatif des actions localisées grains d'abrasif/pièce avec les variables « process » impliquées (durée de toilage, fréquence d'oscillation). Afin de s'affranchir des dispersions des valeurs de la rugosité et de ne pas se contenter de caractériser l'état de surface par un nombre, un critère spécifique a été adopté. Il s'agit du gain de rugosité $G_{R}$ qui est une expression qualitative des effets des variables « process $» . G_{R}$ est défini comme étant le rapport du critère de rugosité $R$ (rugosité mesurée conformément à la norme NF EN ISO 12085 [8]) avant et après l'opération de superfinition.

Le gain de rugosité $G_{R}$ en fonction de la durée de toilage $t_{\text {toilage }}$ est reporté en figure 7 . Dans un premier temps, la dimension des irrégularités de surface diminue considérablement avec la durée de toilage $\left(G_{R}=5,1\right.$ dans les 9 premières secondes). La rugosité $R$ obtenue est de l'ordre de $0,25 \mu \mathrm{m} . G_{R}$ se stabilise à ce niveau au-delà 


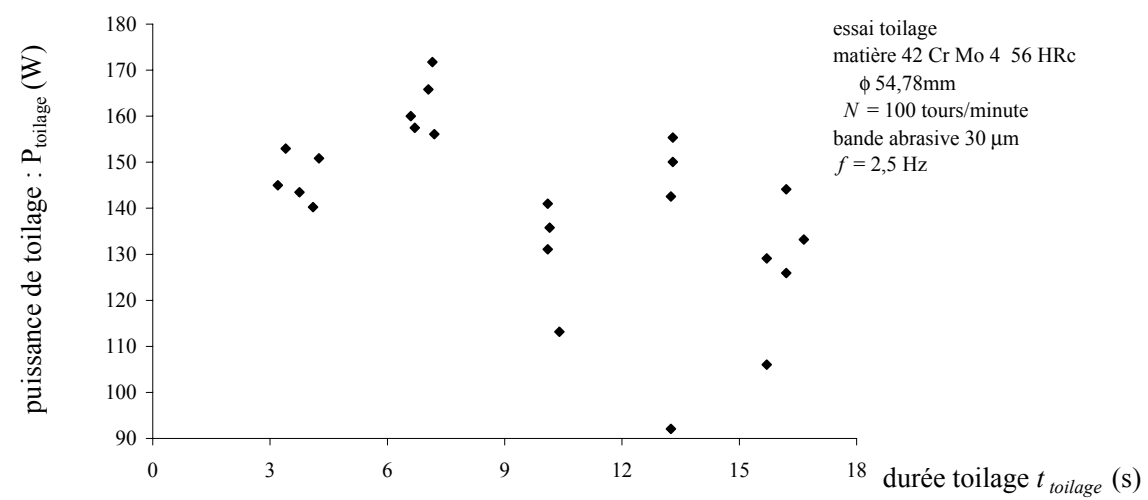

Fig. 5. Détail des puissances de toilage $P_{\text {toilage }}$ pour chaque essai en fonction de la durée du toilage $t_{\text {toilage }}$.

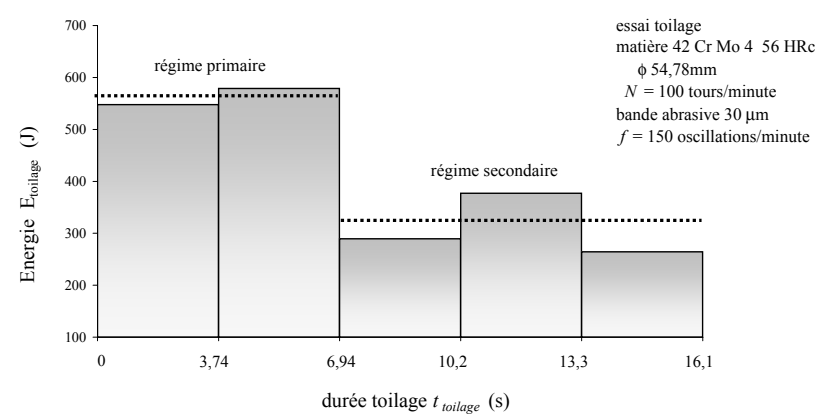

Fig. 6. Évolution de l'énergie de toilage $E_{\text {toilage }}$ en fonction de la durée de l'opération $t_{\text {toilage }}$.

des 9 s de toilage, même si la dispersion augmente sensiblement. Le test statistique d'Aspin-Welch et Student [7] confirme la différence significative au risque de $1 \%$ entre le niveau du gain de rugosité $G_{R}$ pour $t_{\text {toilage }}=3 \mathrm{~s}$ et celui pour $t_{\text {toilage }}=9 \mathrm{~s}\left(t_{\text {student }}=3,32 ; t_{1 \%}=2,95\right)$. Remarquons ici que la variation des irrégularités de surface avec la durée du toilage confirme la présence de deux régimes distincts :

- régime primaire transitoire $\left(G_{R}\right.$ croît);

- régime secondaire établi ( $G_{R}$ constant).

La fréquence d'oscillation influe également (Fig. 8), non seulement sur la diminution du taux des irrégularités de surface de la pièce finie, mais également sur l'activation privilégiée du mécanisme de coupe en toilage. Examinons graphiquement ces deux observations. L'évolution du gain de rugosité montre une progression croissante de $G_{R}$ en fonction de $f$. On note aussi que sans oscillation de la bande abrasive $(f=0)$, le gain de rugosité reste à un niveau très faible de 1,9 par rapport aux fréquences $f$ supérieures. Le test statistique d'AspinWelch et Student [7] confirme la progression significative au risque de $1 \%$ entre le niveau du gain de rugosité $G_{R}$ pour $f=0$ et celui pour $f=0,83 \mathrm{~Hz}\left(t_{\text {student }}=3,71\right.$; $\left.t_{1 \%}=2,98\right)$. Cette augmentation du gain de rugosité $G_{R}$ dès que $f>0$ montre l'influence très importante de l'oscillation transversale de la bande abrasive sur l'action des grains d'abrasifs. En effet, en faisant osciller le contact toile/pièce, la densité de grains actifs augmente.

\section{b) Morphologie des microcopeaux}

La figure 9 résume les observations expérimentales de l'évolution morphologique des micro-copeaux générés en toilage. Pour caractériser cette évolution, une technique de préparation de la toile abrasive a été adoptée. Celle-ci consiste à appliquer un adhésif sur la bande abrasive en fin de toilage afin de décoller la couche ultime de microcopeaux. Cela permet d'accéder aux micro-copeaux primaires, c'est-à-dire ceux formés en début de toilage. Ainsi, cette procédure montre que la composante de coupe en toilage se manifeste essentiellement dans les premiers instants du processus (Fig. 9a). La taille des micro-copeaux générés en début de toilage et logés dans l'espace intergrains est relativement importante. À l'opposé, en fin de toilage, le microcopeaux sont fins, courts et agglomérés (Fig. 9b).

\section{c) État de l'abrasif}

Une caractéristique importante de la toile observée est constituée par sa morphologie : ceci implique à la fois l'angularité des grains et la distribution de leur orientation géométrique au cours du processus de toilage. Les figures 10 et 11 montrent l'évolution respective de la structure des grains d'abrasif en fonction de la durée de toilage $t_{\text {toilage }}$ et de la fréquence d'oscillation $f$. Les formes micrographiques, reportées aux figures 10 et 11, ont été réalisées au MEB, par visée avec une inclinaison à $5^{\circ}$ par rapport à la surface de la bande abrasive. La bande neuve porte des grains d'abrasif orientés, de taille moyenne $30 \mu \mathrm{m}$, qui présentent à la surface leur meilleure aptitude à la coupe (angularité $\approx 15 \mu \mathrm{m}$ ). De plus l'espacement entre les grains est suffisamment grand pour permettre le logement de microcopeaux. Toutefois, après seulement une courte période de toilage (voir Fig. 10), le pouvoir de coupe diminue (l'angularité chute à environ $8 \mu \mathrm{m})$. Jusqu'à $6 \mathrm{~s}$ de toilage, les arêtes tranchantes restent encore vives (voir Fig. 10). Elles s'émoussent ensuite rapidement. Le paramètre de fréquence d'oscillation ne 


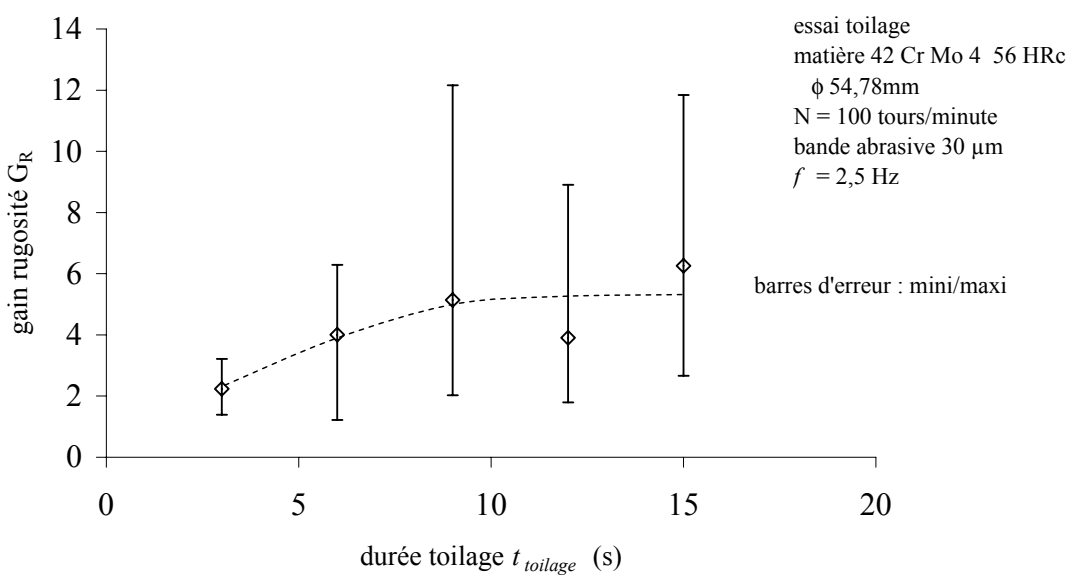

Fig. 7. Évolution du gain de rugosité $G_{R}$ en fonction de la durée de l'opération $t_{\text {toilage }}$.

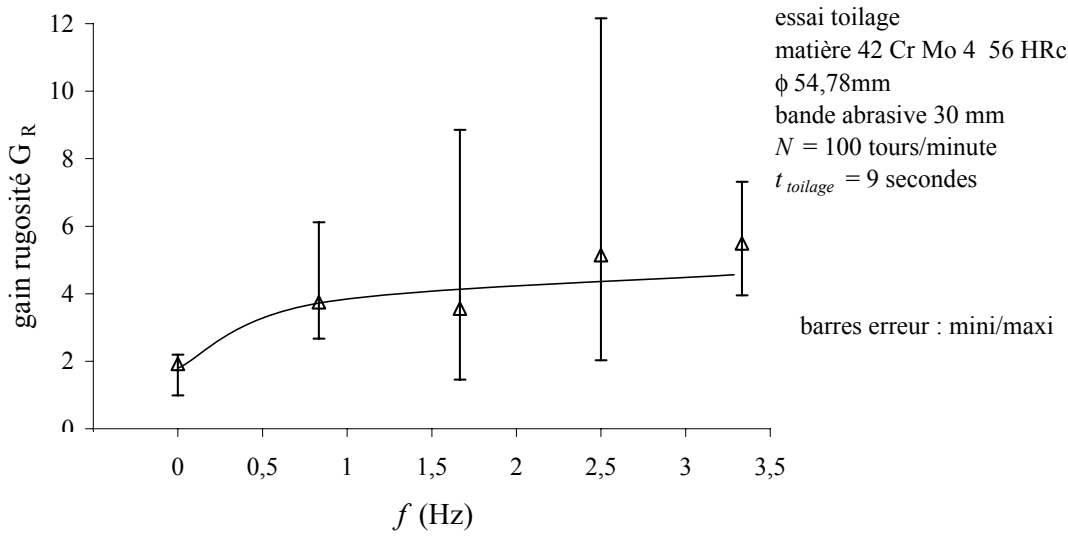

Fig. 8. Évolution du gain de rugosité $G_{R}$ en fonction de la fréquence d'oscillation $f$.

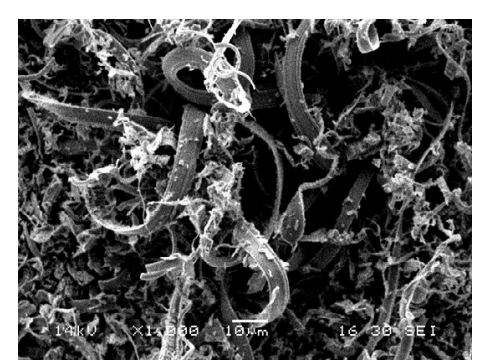

(a) début de toilage

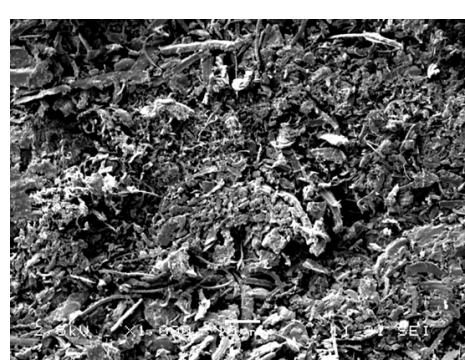

(b) à 9 secondes de toilage

Fig. 9. Morphologie évolutive des micro-copeaux avec la durée de toilage $\left(N=100 \operatorname{tr}^{-m_{n}}{ }^{-1}, f=2,5 \mathrm{~Hz}\right)$.

semble cependant pas avoir d'influence directe et significative sur le mode de fragmentation des grains d'abrasif en toilage (Fig. 11). En effet, l'oscillation de la toile suivant l'axe de la pièce implique que les différents pouvoirs de coupe en bout d'un grain puissent opérer en coupe. Dans ce cas, l'influence de la fréquence d'oscillation se manifeste plus au niveau de l'état de surface que dans la formation de grains broyés qui présentent des morphologies évolutives très marquées : nouvelle angularité ou usure des arêtes vives. En résumé, l'oscillation de la bande augmente le pouvoir de coupe. Cela se traduit par une diminution du $R$ (augmentation de $G_{R}$ ) de la pièce usinée; le paramètre $R$ du film abrasif étant l'image du pouvoir de pénétration d'un grain dans la surface toilée.

\subsection{Discussion}

L'analyse des résultats expérimentaux en fonction de la durée de l'opération de toilage montre deux régimes

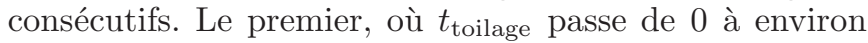
9 s, est caractérisé par une puissance consommée $P_{\text {toilage }}$ relativement importante (jusqu'à 160 watts) et une diminution de la rugosité $R$ dans un rapport $G_{R}$ de 1 à 5,1 . Le second (après $9 \mathrm{~s}$ de toilage) au cours duquel la puissance $P_{\text {toilage }}$ chute rapidement à 130 watts et la rugosité $R$ ne diminue plus de manière significative. Cette évolution est caractéristique de régimes transitoires des phénomènes activés en cours de toilage (phénomènes de coupe, de déformation plastique et de frottement). 


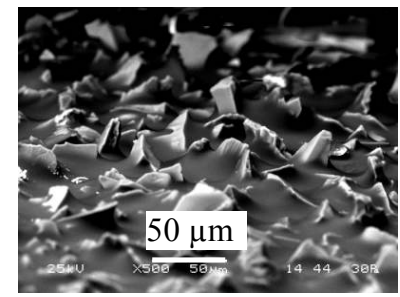

bande neuve

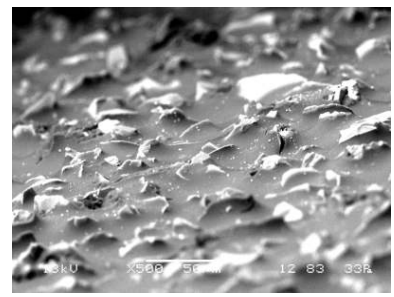

$\mathrm{t}_{\text {toilage }}=9 \mathrm{~s}$

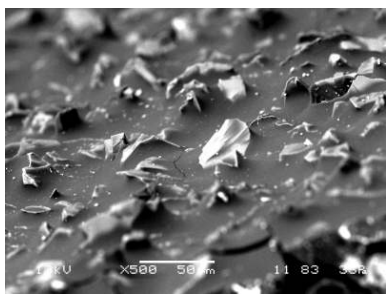

$\mathrm{t}_{\text {toilage }}=3 \mathrm{~s}$

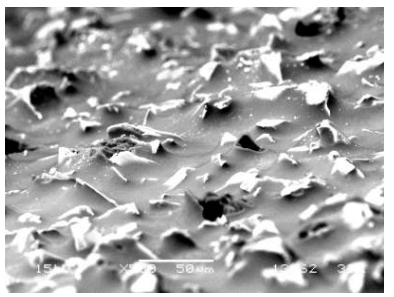

$\mathrm{t}_{\text {toilage }}=12 \mathrm{~s}$

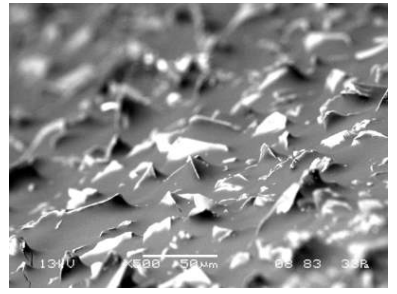

$\mathrm{t}_{\text {toilage }}=6 \mathrm{~s}$

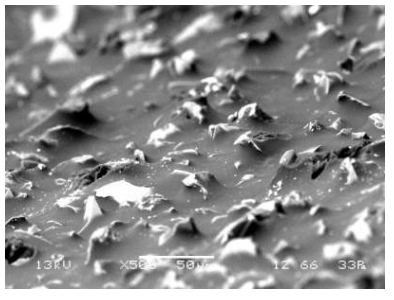

$\mathrm{t}_{\text {toilage }}=15 \mathrm{~s}$

Fig. 10. Morphologie évolutive des grains d'abrasifs en fonction de la durée de toilage $t_{\text {toilage }}\left(N=100\right.$ tr.min $\left.{ }^{-1}, f=2,5 \mathrm{~Hz}\right)$.

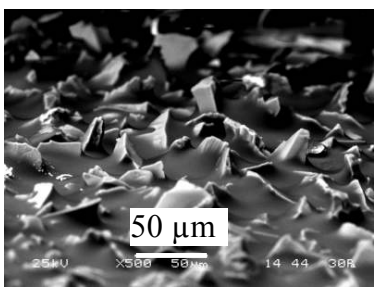

bande neuve

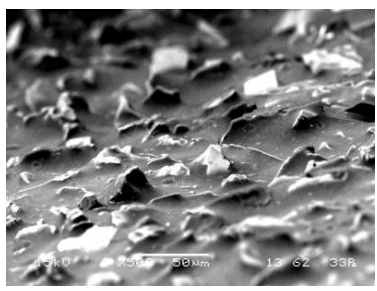

$f=1,67 \mathrm{~Hz}$

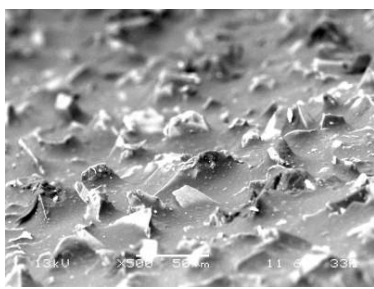

$f=0 \mathrm{~Hz}$

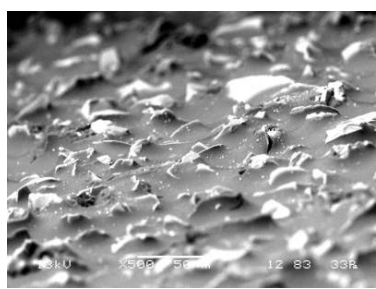

$f=2,50 \mathrm{~Hz}$

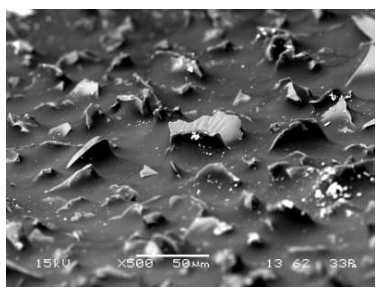

$f=0,83 \mathrm{~Hz}$

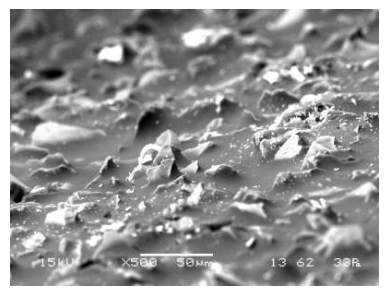

$f=3,33 \mathrm{~Hz}$

Fig. 11. Morphologie évolutive des grains d'abrasifs en fonction de la fréquence d'oscillation $f$. Paramètres de toilage : $N=$ 100 tr. $\min ^{-1}, t_{\text {toilage }}=9 \mathrm{~s}$.

L'observation de la forme des microcopeaux au cours du cycle de toilage et celle des arêtes tranchantes montre une évolution dans le mode de fragmentation des grains abrasifs (voir Figs. 9 et 10). Au début du toilage, le phénomène de coupe est dominant. Les arêtes tranchantes des grains abrasifs sont vives et permettent ainsi la génération de micro-copeaux relativement importants (Fig. 9a). Mais rapidement la hauteur active des grains au-dessus de la résine polymère diminue (voir Fig. 10, durée $6 \mathrm{~s}$ ). Jusqu'à $t_{\text {toilage }}=6 \mathrm{~s}$, les arêtes tranchantes se régénèrent par clivage des grains. Les micro-copeaux deviennent de plus en plus fins. Pour $t_{\text {toilage }}>9 \mathrm{~s}$, les arêtes tranchantes des grains abrasifs s'émoussent (voir Fig. 10). De plus, les micro-copeaux s'agglomèrent en amas entre les grains abrasifs (cf. Fig. 9b) et ne permettent plus à ceux-ci une indentation franche de la surface usinée. Cette formation d'amas de copeaux a pour conséquence la transition accélérée de l'action de coupe à celle du labourage et du frottement sur la surface usinée.

Par ailleurs, la formation de microcopeaux engendrée par la coupe a pour conséquence une diminution rapide de la rugosité de la surface usinée et une prise d'énergie importante (voir Figs. 5 et 7 ). Il faut néanmoins souligner que l'amélioration de la rugosité de surface au cours du régime primaire est considérablement plus faible en l'absence d'oscillation relative bande abrasive/pièce toilée (voir Fig. 8). Cette oscillation permet donc un rendement accru de l'action des grains abrasifs et améliore la formation de microcopeaux.

Le phénomène de frottement s'amplifie au fur et à mesure de l'usure des grains abrasifs. Il est accéléré par les amas de copeaux logés entre les grains qui ainsi empêchent 
l'indentation de surface usinée. Le frottement devient dominant. À ce stade, la puissance consommée chute et se stabilise à un niveau plus faible que celui nécessaire pour générer des microcopeaux (pendant la coupe) ou déformer plastiquement la matière. De plus, ce frottement n'améliore plus la rugosité de surface. Au contraire, le labourage résiduel sans formation de micro-copeaux augmente les dispersions des gains de rugosité (voir Fig. 7).

\subsection{Conclusions}

De cette étude des effets des variables process de toilage (temps de cycle et fréquence d'oscillation du contact film abrasif/pièce), il ressort que la mise au point des tests de qualification du processus de toilage peut être effectuée à partir de mesures énergétiques. Les résultats du test énergétique précisent que

- l'influence du temps de toilage sur la prédominance de chaque composante physique dans la finition de surface fait apparaître deux régimes,

- d'une part, un régime primaire de toilage, qualifié de transitoire, où la superfinition de courte durée implique plutôt une dominance de coupe,

- d'autre part, le prolongement du toilage jusqu'à un régime établi, régime secondaire de toilage, qui conduit à la prédominance de la composante de frottement,

- par oscillation haute fréquence de la bande, il y a diminution du frottement à l'interface grains d'abrasif/microcopeau et un accroissement du pouvoir de coupe.
Par ailleurs, l'avantage de la mesure énergétique est qu'elle est directement applicable sur les machines de production.

Remerciements. Nous remercions le soutien financier de RENAULT/Direction Ingénierie Process (convention CIFRE $\left.\mathrm{n}^{\circ} 769 / 2004\right)$. Les auteurs remercient également les partenaires de l'étude : URT - Usinage de pièces mobiles de Renault.

\section{Références}

[1] M.C. Shaw, Principles of Abrasive Processing, Oxford Science publications, 1996

[2] T.K. Puthanangary, S. Malkin, Experimental investigation of the superfinishing process, Wear 185 (1995) 173-182

[3] B. Varghese, S. Malkin, Experimental investigation of methods to enhance stock removal for superfinishing, Annals of the CIRP 47 (1998) 231-234

[4] S.H. Chang, T.N. Farris, S. Chandrasekar, Contact mechanics of superfinishing, ASME 122 (2000) 388-393

[5] B. Varghese, S. Malkin, A selection of optimal superfinishing parameters, Rapport Technique MR99-249, SME, 1999

[6] S.H. Chang, T.N. Farris, S. Chandrasekar, Experimental characterization of superfinishing, IMechE, 217 (2000) 941-951

[7] AFNOR, «NF-X-06-050 : Étude de la normalité d'une distribution », Norme AFNOR, 1991

[8] AFNOR, «NF-EN-ISO-12085 : État de surface : Méthode du profil, Paramètres liés au profil », Norme AFNOR, 1998 